EPJ manuscript No.

(will be inserted by the editor)

\title{
Fermionic renormalization group flow into phases with broken discrete symmetry: charge-density wave mean-field model
}

\author{
Roland Gersch ${ }^{1}$, Carsten Honerkamp ${ }^{2}$, Daniel Rohe ${ }^{1}$, and Walter Metzner ${ }^{1}$ \\ 1 Max-Planck-Institut für Festkörperforschung, Heisenbergstraße 1, D-70569 Stuttgart, Germany \\ ${ }^{2}$ Institut für Theoretische Physik, Universität Würzburg, Am Hubland, D-97074 Würzburg, Germany \\ Submitted 9 September 2005, published online 23 December 2005
}

\begin{abstract}
We generalize the application of the functional renormalization group (fRG) method for the fermionic flow into the symmetry-broken phase to finite temperatures. We apply the scheme to the case of a broken discrete symmetry: the charge-density wave (CDW) mean-field model at half filling. We show how an arbitrarily small initial CDW order parameter starts to grow at the CDW instability and how it flows to the correct final value, suppressing the divergence of the effective interaction in the fRG flow. The effective interaction peaks at the instability and saturates at low energy scales or temperatures. The relation to the mean-field treatment, differences compared to the flow for a broken continuous symmetry, and the prospects of the new method are discussed.
\end{abstract}

PACS. 71.45.Lr, 71.10.Fd Charge-density-wave systems, Lattice fermion models (Hubbard model, etc).

\section{Introduction}

Renormalization group (RG) techniques are a standard tool for the analysis of the low-energy physics of interacting fermions [1]2,3. Although new effective degrees of freedom may be generated in the RG flow down to lower energies, perturbative RG flows with the initial degrees of freedom - in our case electrons - are considered the least biased approaches to the variety of competing and conspiring tendencies at low scales. Without appropriate self-energy corrections these flows often diverge at ' a nonzero energy scale, and not all fermionic modes can be integrated out. These divergences are signatures of potential phase transitions, typically involving some kind of symmetry breaking. Hence, these flows still contain useful physical information. In many cases the physics below ' the critical scale can be explored by other means, e.g. by a mean-field treatment [4, by a bosonic description [5] or by exact diagonalization of a restricted low-energy Hamiltonian 6]. Yet, the latter methods always require some sort of simplification of the low-energy action, and typically the final results depend on the scale where the transition from one treatment to the other is performed. Therefore, it is desirable to have an RG scheme which can be continued beyond the noted divergence and which allows a controlled access to the low-energy phase.

Recently, Salmhofer et al. [7] have proposed an extension of the fermionic functional RG flow into the symmetrybroken regime. The key idea is to include an arbitrarily small symmetry breaking field, which we include in the RG as a symmetry-breaking component in the initial condition for the fermionic self-energy. This component grows rapidly at the critical scale and prevents a true divergence of the interactions. Therefore, the flow can be continued down to the lowest scales. Spontaneous symmetry breaking is captured by sending the external symmetrybreaking field to zero after integrating the flow. For meanfield models like the reduced BCS model, this procedure yields the exact solution already within a suitably chosen one-loop truncation of the vertex flow [7].

Of course this method should now be extended to other models and other physical situations. In particular, the hope is that non-mean-field models like the two-dimensional Hubbard model will be treatable. There, the method would yield the flow into symmetry-broken phases corresponding to correlations for which the initial interaction does not contain an attractive component and where the attraction is generated during the flow. For example, this is the case for $d$-wave pairing in the Hubbard model on the two-dimensional square lattice near half filling. Furthermore, the non-mean-field parts of the interactions would not need to be dropped and their influence could be studied.

Before proceeding to such more complicated problems, we extend the application of the formalism to nonzero temperatures in the context of the charge-density wave (CDW) mean-field model on a $d$-dimensional hypercubic lattice at half band filling. Again, we closely monitor the correspondence to mean-field theory. This example is interesting because, in contrast to the BCS model studied in Ref. [7, only a discrete symmetry is broken. Hence we learn about the differences between the RG flows for broken continuous and discrete symmetries. 
Gersch, Honerkamp, Rohe, Metzner: RG flow into CDW state

This paper is organized as follows. In Sec. 2 we introduce the CDW model which we analyze in the remainder. In Sec. 3 we present an exact solution in the thermodynamic limit employing a resummation of perturbation theory. We briefly introduce the renormalization group technique in Sec. 4 and write down the functional RG equations for the self-energy and the effective interaction. In Sec. 5. these equations are discussed in the case of vanishing temperature, where they can be presented in a considerably simpler way. The finite-temperature equations are treated numerically in Sec. 6. Conclusions and outlook are presented in Sec. []

\section{Model}

We consider spinless fermions on a $d$-dimensional hypercubic lattice with $N$ sites labeled by $\boldsymbol{x}$. The kinetic energy shall be given by a tight-binding dispersion with nearestneighbor hopping amplitude $t$. Further we assume a repulsive nearest-neighbor density-density interaction $V_{0}$. Then, the Hamiltonian reads

$$
H=-t \sum_{\boldsymbol{x}, \boldsymbol{n}}\left(c_{\boldsymbol{x}}^{\dagger} c_{\boldsymbol{x}+\boldsymbol{n}}+h . c .\right)+V_{0} \sum_{\boldsymbol{x}, \boldsymbol{n}} c_{\boldsymbol{x}}^{\dagger} c_{\boldsymbol{x}} c_{\boldsymbol{x}+\boldsymbol{n}}^{\dagger} c_{\boldsymbol{x}+\boldsymbol{n}}
$$

The sum over $\boldsymbol{n}$ runs over the $d$ unit vectors of the $d$ dimensional hypercubic lattice with lattice constant set to one. Now we assume half filling with an average of one particle per two lattice sites, $\left\langle n_{\boldsymbol{x}}\right\rangle=1 / 2$. In this case it is easy to see that the nearest-neighbor density-density repulsion will favor a charge density wave (CDW), i.e. a periodic arrangement of the particles in which the probability of two particles being nearest neighbors is reduced. This tendency competes with the hopping term of the kinetic energy and with the entropy, which are minimized and maximized, respectively, for an equal population of all sites. In the case where a CDW is formed with a fixed modulation amplitude $n_{\mathrm{CDW}}$, the charge density $\left\langle n_{\boldsymbol{x}}\right\rangle$ takes only two values, $1 / 2 \pm n_{\mathrm{CDW}}$, on the two inter-penetrating sublattices with doubled unit cells. Therefore, the halffilled CDW state breaks a discrete Ising-type symmetry, and the degeneracy of the ordered state is twofold. While in one dimension such order can only occur in the ground state, in two and higher dimension it is possible to have a nonzero critical temperature $T_{c}$.

For general band filling, a CDW with an incommensurate modulation wave-vector generates infinitely many different values of average populations on the lattice sites. This corresponds to an arbitrary phase of the density modulation at an arbitrary reference lattice site. Hence in the incommensurate case the CDW breaks a continuous symmetry.

Continuing with the half-filled case, we Fourier-transform the Hamiltonian using

$$
c_{\boldsymbol{x}}=\frac{1}{\sqrt{N}} \sum_{\boldsymbol{k}} e^{i \boldsymbol{k} \boldsymbol{x}} c_{\boldsymbol{k}}
$$

and obtain

$$
\begin{aligned}
H= & -2 t \sum_{\boldsymbol{k}} L(\boldsymbol{k}) c_{\boldsymbol{k}}^{\dagger} c_{\boldsymbol{k}} \\
& +\frac{V_{0}}{N} \sum_{\boldsymbol{k}_{1}, \boldsymbol{k}_{2}, \boldsymbol{q}} L(\boldsymbol{q}) c_{\boldsymbol{k}_{1}}^{\dagger} c_{\boldsymbol{k}_{1}-\boldsymbol{q}} c_{\boldsymbol{k}_{2}}^{\dagger} c_{\boldsymbol{k}_{2}+\boldsymbol{q}}
\end{aligned}
$$

Here we have introduced $L(\boldsymbol{k})=\sum_{i=1}^{d} \cos \left(k_{i}\right)$. Abbreviating the dispersion as $\xi(\boldsymbol{k})=-2 t \sum_{i=1}^{d} \cos \left(k_{i}\right)=-2 t L(\boldsymbol{k})$, we note that the nesting condition $\xi(\boldsymbol{k})=-\xi(\boldsymbol{k}+\boldsymbol{Q})$ is fulfilled for the wave-vector $\boldsymbol{Q}=(\pi, \ldots, \pi)$. This causes a divergence of the non-interacting charge response at the nesting wave-vector for $T \rightarrow 0$. If the interactions are treated in the random phase approximation, the charge response at $\boldsymbol{Q}$ will actually diverge at a finite temperature, giving an estimate of $T_{c}$ for the charge-density wave formation. In a mean-field treatment of the symmetry-breaking in which the interaction term is decoupled with an alternating charge density, anomalous particle-hole pairing expectation values $\left\langle c_{\boldsymbol{k}+\boldsymbol{Q}}^{\dagger} c_{\boldsymbol{k}}\right\rangle$ will become nonzero below the transition temperature.

Although this physical picture of a CDW transition is essentially correct regarding the ground state properties, the model (3) cannot be solved exactly. This changes if we reduce the interactions by only keeping processes that change both particle's momenta by $\boldsymbol{Q}$,

$$
\begin{aligned}
H_{\mathrm{red}}= & \sum_{\boldsymbol{k}} \xi(\boldsymbol{k}) c_{\boldsymbol{k}}^{\dagger} c_{\boldsymbol{k}} \\
& -\frac{V_{0}}{N} \sum_{\boldsymbol{k}_{1}, \boldsymbol{k}_{2}} c_{\boldsymbol{k}_{1}}^{\dagger} c_{\boldsymbol{k}_{1}-\boldsymbol{Q}} c_{\boldsymbol{k}_{2}}^{\dagger} c_{\boldsymbol{k}_{2}+\boldsymbol{Q}}
\end{aligned}
$$

Now the interaction between fermions on the lattice sites $\boldsymbol{x}$ and $\boldsymbol{x}^{\prime}$ corresponds to an infinite range density-density interaction with oscillating sign,

$-V_{0} N^{-1} \cos \left[\boldsymbol{Q}\left(\boldsymbol{x}-\boldsymbol{x}^{\prime}\right)\right] n_{\boldsymbol{x}} n_{\boldsymbol{x}^{\prime}}$

Analogous to the reduced BCS pairing model [8, in the reduced model (4) mean-field theory becomes exact in the thermodynamic limit $N \rightarrow \infty$ and there is a CDW ordered state below a critical $T_{c}>0$ in any dimension $d$. For the half-filled commensurate case, the CDW state is twofold degenerate and the electronic order parameter $n_{\mathrm{CDW}}$ is real.

Next we include a real external field $\Delta_{\text {ext }} \cos (\boldsymbol{Q} \boldsymbol{x})$. This term breaks the translational symmetry of the original Hamiltonian explicitly and lifts the degeneracy of the two CDW ground states. In momentum space this term couples to pairs of particles whose momenta differ by $\boldsymbol{Q}=(\pi, \ldots, \pi)$. If $\Delta_{\text {ext }}$ is very small compared to all other relevant energy scales, it does not change macroscopic observables away from the critical temperature. However, it allows us to integrate the RG differential equations over all scales without encountering divergences. The Hamiltonian including the external field reads

$$
\begin{aligned}
H_{\mathrm{red}}= & H_{\mathrm{kin}}+\sum_{\boldsymbol{k}} \Delta_{\mathrm{ext}} c_{\boldsymbol{k}}^{\dagger} c_{\boldsymbol{k}+\boldsymbol{Q}} \\
& -\frac{V_{0}}{N} \sum_{\boldsymbol{k}_{1}, \boldsymbol{k}_{2}} c_{\boldsymbol{k}_{1}}^{\dagger} c_{\boldsymbol{k}_{1}+\boldsymbol{Q}} c_{\boldsymbol{k}_{2}}^{\dagger} c_{\boldsymbol{k}_{2}-\boldsymbol{Q}} .
\end{aligned}
$$


We introduce a frequency-space field-integral representation in the usual way. Writing $T$ for temperature, we define the fermionic Matsubara frequencies $\omega_{j}:=(2 j+1) \pi T$ and obtain Grassmann fields $\psi_{\boldsymbol{k}, \omega_{n}}, \bar{\psi}_{\boldsymbol{k}, \omega_{n}}$. Introducing the Nambu-like notation

$$
\begin{gathered}
\Psi_{\boldsymbol{k}, \omega_{n}}=\left(\begin{array}{c}
\psi_{\boldsymbol{k}, \omega_{n}} \\
\psi_{\boldsymbol{k}+\boldsymbol{Q}, \omega_{n}}
\end{array}\right), \\
\bar{\Psi}_{\boldsymbol{k}+\boldsymbol{Q}, \omega_{n}}=\left(\bar{\psi}_{\boldsymbol{k}, \omega_{n}} \bar{\psi}_{\boldsymbol{k}+\boldsymbol{Q}, \omega_{n}}\right),
\end{gathered}
$$

the partition function reads

$$
\begin{gathered}
\mathcal{Z}=\int \mathcal{D}(\bar{\psi}, \psi) \exp \left[\frac{1}{2} T \sum_{n, \boldsymbol{k}} \bar{\Psi}_{\boldsymbol{k}, \omega_{n}} \mathbb{Q}\left(\xi_{\boldsymbol{k}}, \omega_{n}\right) \Psi_{\boldsymbol{k}, \omega_{n}}\right. \\
\left.+V_{0} \frac{T^{3}}{N} \sum_{\substack{n_{1}, n_{2}, n_{3} \\
\boldsymbol{k}_{1}, \boldsymbol{k}_{2}}} \bar{\psi}_{\boldsymbol{k}_{1}, \omega_{n_{1}}} \psi_{\boldsymbol{k}_{1}+\boldsymbol{Q}, \omega_{n_{3}}} \bar{\psi}_{\boldsymbol{k}_{2}, \omega_{n_{2}}} \psi_{\boldsymbol{k}_{2}-\boldsymbol{Q}, \omega_{4}}\right] .
\end{gathered}
$$

Here, $\omega_{4}=\omega_{n_{1}+n_{2}-n_{3}}$ and $\mathbb{Q}$ is the matrix inverse of the free Green's function (see e.g. [9])

$$
\mathbb{G}_{0}\left(\xi, \omega_{n}\right)=\frac{1}{\omega_{n}^{2}+\xi^{2}+\Delta_{\mathrm{ext}}^{2}}\left(\begin{array}{cc}
-i \omega_{n}-\xi & -\Delta_{\mathrm{ext}} \\
-\Delta_{\mathrm{ext}} & -i \omega_{n}+\xi
\end{array}\right)
$$

We have dropped the momentum argument of $\xi$ for brevity. The minus sign in front of the first $\xi$ arises from the $\boldsymbol{Q}$ anti-periodicity of the cosine. The doubling of the number of degrees of freedom by introducing the Nambu notation is compensated by the $1 / 2$ in front of the quadratic part of the action. Alternatively, we could integrate over only half the Brillouin zone. Due to the explicit symmetry breaking, there is a nonzero off-diagonal component in the propagator. If we apply mean-field theory by decoupling the interaction term in the CDW channel, the CDW order parameter due to the interaction, $\Delta_{\text {ia }}$, will add to $\Delta_{\text {ext }}$.

\section{Mean-Field Solution and Resummation}

In this section, we resum the perturbation expansions for the self-energy and the effective interaction in the thermodynamic limit $N \rightarrow \infty$. For our model this is equivalent to mean-field theory in the CDW amplitude with modulation wavevector $\boldsymbol{Q}$.

Due to the explicit symmetry breaking with $\Delta_{\text {ext }} \neq 0$ there is a nonzero frequency independent off-diagonal selfenergy which scatters a fermion with wavevector $\boldsymbol{k}$ to $\boldsymbol{k}+\boldsymbol{Q}$ and vice versa. Therefore, besides normal (diagonal) wave-vector conserving propagators, also anomalous (off-diagonal) propagators appear in the diagrams of the perturbation theory. As indicated above, compared to the non-interacting propagator in (7), the effect of the interactions is that we have to supplement $\Delta_{\text {ext }}$ with an offdiagonal self-energy $\Delta_{\text {ia }}$ caused by the interaction. In the following we determine its value.

Since the interaction term in (5) includes a prefactor $1 / N$, only diagrams having one summation over all degrees (a)

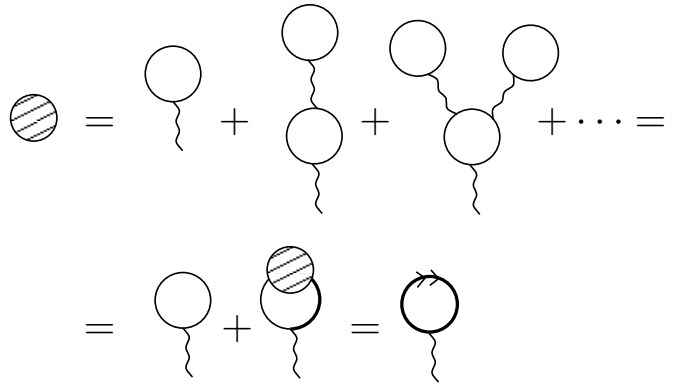

(b)

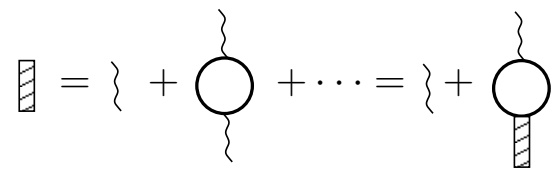

Fig. 1. Resummation of the perturbation expansion of the reduced charge-density-wave model: (a) self-energy (hatched circle), and (b) effective interaction (hatched rectangle). Undirected internal lines carry a summation over their Nambu-like indices in addition to the frequency summation and momentum integration. Double-arrowed lines correspond to off-diagonal propagator elements, bold lines to full and thin lines to bare propagators.

of freedom per interaction line contribute in the thermodynamic limit. This together with the special structure of the interaction entails that only the bubble diagrams shown in Fig. 1(b) contribute to the perturbation series for the effective interaction in second order of the bare interaction. In all other second order diagrams the restricted interaction implies that the internal momentum is fixed by an external momentum. Then the contribution of these diagrams is $O(1 / N)$ and vanishes for $N \rightarrow \infty$. Furthermore, since each bubble in the remaining diagrams transfers a momentum of $\boldsymbol{Q}$ due to the special structure of the bare interaction, only effective interaction processes with a momentum transfer of $\boldsymbol{Q}$ exist. This causes the normal diagonal self-energy to vanish as well. These arguments can be iterated to arbitrary order, and hence only the bubble chains indicated in Fig. 1(b) contribute to the perturbation expansion of the effective interaction. In particular, no anomalous effective interactions which violate the original translation symmetry are generated. This is different away from half-filling where pairs of internal anomalous propagators can generate effective interactions which violate momentum conservation by adding even multiples of the modulation wavevector $Q$ to their incoming total momentum. In the half-filled case $2 \boldsymbol{Q}=(2 \pi, \ldots, 2 \pi)$ is a reciprocal lattice vector, and therefore no new effective interactions appear.

In Fig. 11(a) we show the perturbation expansion for the off-diagonal self-energy. Again, due to the special structure of the interaction only those diagrams which retain one momentum integration per interaction line contribute to the perturbation expansion of the self-energy in the thermodynamic limit. Furthermore, the interaction is nonzero only for momentum transfer $\boldsymbol{Q}$. Thus, in the thermodynamic limit only diagrams constructed from tadpole diagrams contribute. If a tadpole diagram is connected to 
at most one other tadpole diagram, only the terms offdiagonal in the Nambu-like space are non-vanishing. This is because the outgoing momentum of such an off-diagonal line equals the incoming momentum plus $\boldsymbol{Q}$. The exact resummation is equivalent to self-consistent Hartree-Fock theory of which the Fock contributions vanish in the thermodynamic limit. Another way of saying this is that Hartree mean-field theory is exact in this model.

In the following, we assume the density of states to be constant over the Brillouin zone and equal to its value at the Fermi energy to remove dimension-specific effects. Evaluating Fig. M(a) and denoting the self-energy due to the interaction by $\Delta_{\mathrm{ia}}$, the band edge by $W$, the density of states at the Fermi energy $\rho_{0}, \Sigma:=\Delta_{\text {ia }}+\Delta_{\text {ext }}$ as well as $E:=\sqrt{\xi^{2}+\Sigma^{2}}$, we obtain the gap equation (compare e.g. [10])

$$
\Sigma-\Delta_{\text {ext }}=V_{0} \Sigma \rho_{0} \int_{0}^{W} \mathrm{~d} \xi \frac{\tanh (E / 2 T)}{E}
$$

which resembles the BCS [11] and excitonic insulator [12] gap equations. If we set $T=\Delta_{\text {ext }}=0$, solving (8) analytically yields (compare e.g. [11,13])

$$
\Sigma=2 t \frac{1}{\sinh \left(1 / V_{0} \rho_{0}\right)} \stackrel{V_{0} \rho_{0} \ll 1}{\approx} 4 t \exp \left(-\frac{1}{V_{0} \rho_{0}}\right) .
$$

Similar equations are found in the BCS theory for superconductors [11.

We now turn to the effective interaction with zero frequency transfer. As argued above, only fermionic bubbles contribute and all other diagrams vanish in the thermodynamic limit $N \rightarrow \infty$. In the notation, we omit the arguments of the hyperbolic functions originating from the Fermi distribution from now on: they are always $E / 2 T$. Abbreviating the bubble integral

$$
B:=\rho_{0} \int_{0}^{W} \frac{\mathrm{d} \xi}{E^{2}}\left[\frac{\Sigma^{2}}{2 T} \cosh ^{-2}+\frac{\xi^{2}}{E} \tanh \right]
$$

the evaluation of Fig. प(b) yields

$$
V=\frac{V_{0}}{1-V_{0} B}
$$

If $\Delta_{\text {ext }}=0$, we can define the critical temperature $T_{c}$ via the condition that $1=V_{0} B\left(T_{c}\right)$, i.e. that the denominator of (11) vanishes and $V$ diverges. By analyzing (10) we note that this criterion for $T_{c}$ coincides with the temperature below which we can find a nonzero solution of the gap equation (8). For small $V_{0}$ and $\Sigma=0$ we find $1.76 T_{c} \approx$ $4 t \exp \left(-1 / V_{0} \rho_{0}\right) \approx \Sigma(T=0)$ (see e.g. [13]). This is true when the approximation in (9) is valid.

As a finite $\Delta_{\text {ext }}$ will later allow us to integrate the renormalization group equations over all scales, we are interested in the dependence of the mean-field solutions [8] and (11) on $\Delta_{\text {ext }}$. In particular, we will illustrate where the system including a finite symmetry-breaking field (5) is practically indistinguishable from the system without symmetry-breaking field, (44). To this end, we show in the
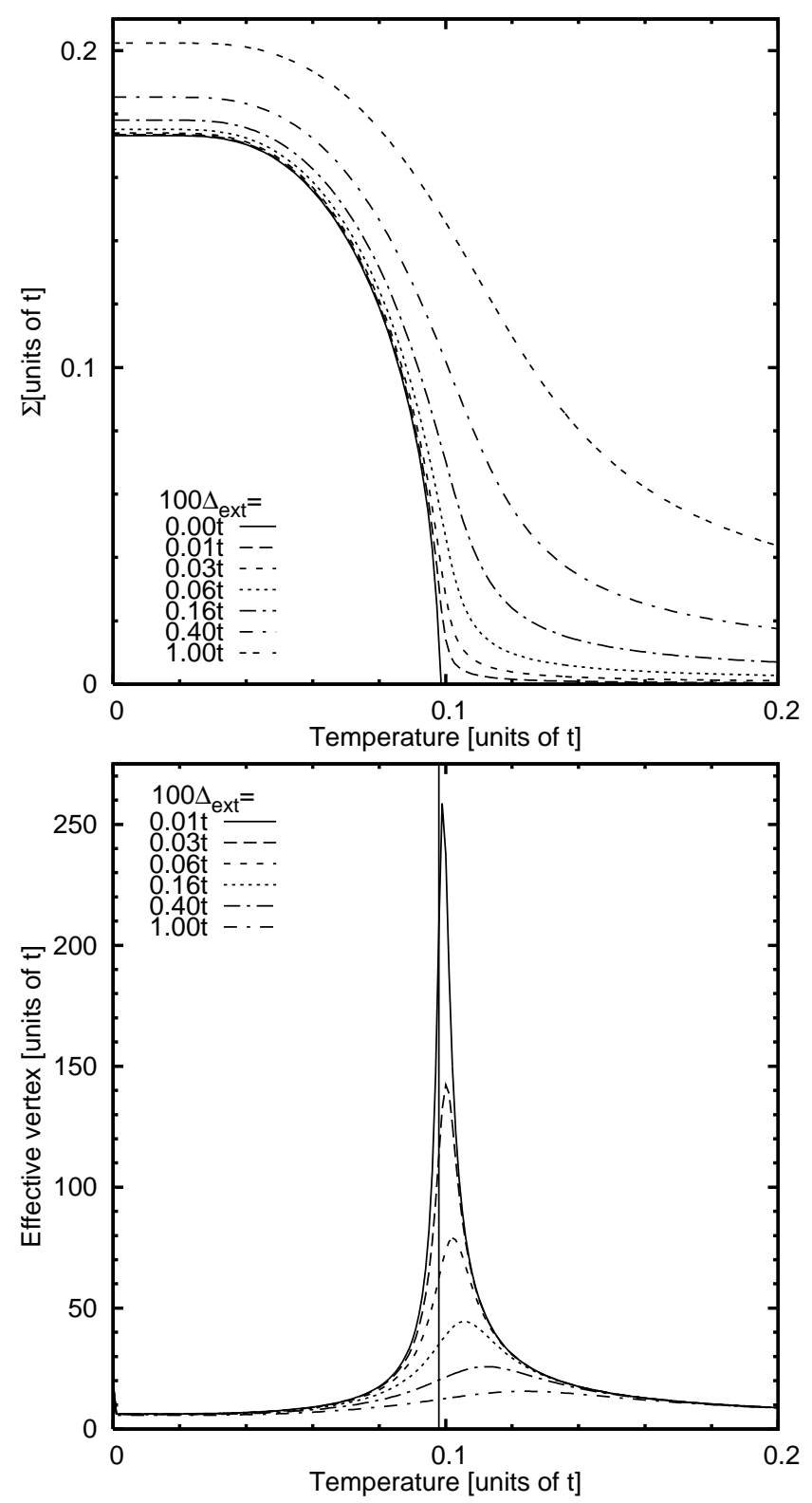

Fig. 2. (top) Solutions $\Sigma(T)$ of the gap equation for $V_{0}=2 t$ and small to intermediate initial self-energies $\Delta_{\text {ext }}$. (bottom) Temperature dependence of the effective interactions calculated by resumming the perturbation expansion for $V_{0}=2 t$ and small to intermediate initial self-energy. $\rho_{0}=1 / 2 \pi$ for all numerical calculations.

upper part of Fig. 2] convergence of $\Sigma^{\Delta_{\text {ext }}}(T)$ to $\Sigma(T)$ for $\Delta_{\text {ext }} \rightarrow 0$. The convergence is worst in the vicinity of a kink that can be discerned at $T \approx 0.1 t$ in the graph for $\Delta_{\text {ext }}=0$. We would like to be more concrete about the temperature range in which the system including a symmetry-breaking field can be used to approximate the system without symmetry-breaking field. To determine more precisely the temperature range in which the symmetry-breaking field has little impact on the physics of the system, we consider the dependence of the effective interaction on both temperature and the symmetry- 
breaking field as plotted in the lower part of Fig. 2] Its singularity is regularized by a nonzero $\Delta_{\text {ext }}$ which cuts off the integrand of the bubble integral (10). The figure also shows a strong suppression of the effective interaction peak with increasing $\Delta_{\text {ext }}$ (the dependence of the peak height on $\Delta_{\text {ext }}$ is plotted in Fig. (7). Its location converges to $T_{c}$ from above for $\Delta_{\text {ext }} \rightarrow 0$. In the figure, we also spot convergence of $V^{\Delta_{\text {ext }}}(T)$ to $V(T)$ for $T \ll T_{c}$ and $T \gg T_{c}$. We see that $V^{\Delta_{\operatorname{ext}}}(T) \approx V(T)$ if $T$ is outside the double width at half maximum of $V^{\Delta_{\text {ext }}}(T)$. If $T$ is outside said width and also below $T_{c}$, we learn from the upper part of the figure that $\Sigma^{\Delta_{\operatorname{ext}}}(T) \approx \Sigma(T)$. This is thus the temperature region in which the initial symmetry-breaking field does not appreciably change the physics.

\section{Renormalization Group Setup}

We will now apply the one-particle irreducible (1PI) functional renormalization group scheme to the charge-densitywave. The scheme is described in 1 (see also 14) and is here employed in the version suggested by Katanin [15. In this version, the differential equations for the self-energy and four-point function constitute a closed system. The special structure of our bare interaction will allow us to further simplify the equations. We will see that in contrast to the BCS flow [7, no anomalous effective interactions are generated. In later sections, we will verify numerically that the $\mathrm{fRG}$ reproduces the resummation results.

Following [1], we introduce a positive real parameter $s$ and a cutoff $\Lambda:=2 t \exp (-s)$. In our calculations, all modes satisfying $|\xi|>\Lambda$ have been integrated out. The effective interaction and self-energy at this scale can thus be interpreted as parameters of an effective theory for a reduced system with smaller bandwidth. We start the flow at $s=0(\Lambda=2 t)$, where all modes have yet to be integrated out, and integrate the flow down to $\Lambda=0$. To analytically implement this procedure, we introduce $\chi(\xi, \Lambda)$ as a placeholder for any cutoff function, which shall have range $[0,1]$, assume the value $1 / 2$ when $\Lambda=\xi$, approach 0 when reducing $\xi$ below $\Lambda$ and 1 when increasing $\xi$ above $\Lambda$. We replace $\mathbb{Q}(\xi)$ by $\mathbb{Q}(\xi) / \chi(\xi, \Lambda)$, suppressing low-energy modes in the field integral and rendering the self-energy and effective interaction scale-dependent. In the following, dots over symbols will denote differentiation with respect to $s$. In the diagrammatic representations, a heavy line represents a scale-dependent full propagator (calculated with the scale-dependent self-energy), a hatched circle represents the scale-dependent self-energy and a hatched rectangle represents the scale-dependent effective interaction. We furthermore introduce the singlescale propagator $\mathbb{S}:=\mathbb{G} \mathbb{Q} \mathbb{G}$ (all scale-dependent). A line drawn through propagator lines indicates an $s$-derivative.

In the flow of the effective interaction, having $\partial_{s}(\mathbb{G} \mathbb{G})$ 15] instead of $\mathbb{S} \mathbb{G}+\mathbb{G} \mathbb{S}$ as in the original $1 \mathrm{PI} R \mathrm{R}$ scheme 1 is crucial in order to be able to follow the flow down to $\Lambda=0$. In the Katanin version of the $1 \mathrm{PI}$ scheme, the exact hierarchy of RG differential equations with the exception of the first equation is written using only full fourpoint functions, full propagators and scale-differentiated full propagators on the right-hand sides. The differential equations for the self-energy and the four-point function thus constitute a closed system. In particular, the contribution to the flow of the four-point function which involves the six-point function in the standard 1PI scheme [1] is taken into account in the Katanin scheme by diagrams involving only four-point functions and full or scaledifferentiated full propagators, respectively. The part with overlapping loops of this contribution vanishes for the special structure of the interaction and the thermodynamic limit considered here. The remaining part of this contribution is taken into account by the replacement of the singlescale propagator by the scale-differentiated full propagator. Hence, the graphical form of the fRG differential equations is as shown in Fig. 3 ]

In the flow of the effective interaction (right part of Fig. 3), the summation over the 'Nambu' indices of the internal lines includes normal and anomalous propagators. Analogous to the perturbation expansion in the previous section, in the limit $N \rightarrow \infty$ the special momentum structure of the initial interaction is conserved by the RG flow of the effective interaction. In the present half-filled case, no new effective interactions with different external legs are generated. This is in contrast to the BCS pairing model, where U(1)-symmetry-breaking vertices with four incoming or four outgoing legs are generated. Linear combinations of normal and anomalous components of the effective interacion could be identified with 'amplitude' and 'phase' vertices. In the half-filled CDW model the effective interaction behaves like the 'amplitude' vertex of the BCS problem, while the 'phase' vertex is missing as there is no breaking of a continuous symmetry. The self-energy only contains the off-diagonal part of the single-scale propagator, again due to the restricted form of the interaction. For a derivation see [1] and 17. Ref. 7] also provides an analytical proof of the equivalence of the flow equations (Fig. 3) and the resummation equations (Fig. 11).

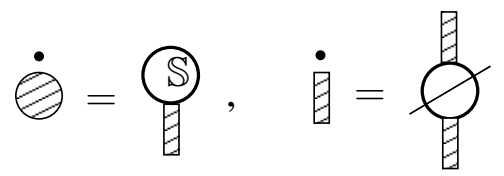

Fig. 3. The hierarchy of flow equations for the self-energy and the effective interaction.

Evaluating the diagrams of Fig. [3] we suppress scale and frequency dependence in the notation and write $E:=$ $\sqrt{\xi^{2}+\chi^{2} \Sigma^{2}}$. The RG differential equations now read

$$
\begin{gathered}
\dot{V}=\rho_{0} V^{2}\left\{\left.\frac{\Lambda}{E}\left(\frac{\Lambda^{2}}{E^{2}} \tanh +\frac{\Sigma^{2} \beta}{2 E} \cosh ^{-2}\right)\right|_{\substack{\xi=\Lambda \\
\chi=1}}-\right. \\
\int_{\Lambda}^{W} \frac{\mathrm{d} \xi}{E} \frac{\Sigma \dot{\Sigma}}{E^{2}}\left[\frac{3 \xi^{2}}{E^{2}}\left(\tanh -\frac{\beta E}{2} \cosh ^{-2}\right)\right. \\
\left.+\beta^{2} \Sigma^{2} \frac{\tanh ^{2} \cosh ^{2}}{2}\right\}
\end{gathered}
$$




$$
\dot{\Sigma}=\left.V \frac{\Sigma \rho_{0} \Lambda}{E} \tanh \right|_{\substack{\xi=\Lambda \\ \chi=1}},
$$

where we have used $\mathbb{S}=\dot{\chi} \partial \mathbb{G} / \partial \chi$, the limit of a sharp cutoff, and (3.19) of [16] (The simplification of the equations obtained using the limit of a sharp cutoff is due to Tilman Enss). The initial conditions are $\left.\Sigma\right|_{s=0}=\Delta_{\text {ext }}$ and $\left.V\right|_{s=0}=V_{0}$. If we choose $\Delta_{\text {ext }}=0, \Sigma$ remains zero for all scales. Then only the first term in (12) remains, and the flow will diverge at a nonzero scale $\Lambda_{c}$. We would thus be neither able to integrate over all scales nor compare our results to the resummation of section 3 We therefore always study the RG equations for a finite $\Delta_{\text {ext }}$, usually $10^{-4} t$. We will show that we can thereby circumvent the divergence of the effective interaction and still get arbitrarily close to the exact mean-field results without explicit symmetry breaking in the temperature range determined in section 3 ,

\section{Renormalization Group at Zero Temperature}

We first consider zero temperature, $T=0$. In this case, it is easier to write down the analytical expressions, the roles of the different right-hand side terms are easily identifiable, and the value of the effective interaction at $\Lambda=0$ is nearly independent of $\Delta_{\text {ext }}$, as $\Lambda_{c}$ is larger than the double width at half maximum of the effective interaction flow peak (see Fig. 团 lower part).

In the limit $T \rightarrow 0$, (12) and (13) become

$$
\begin{aligned}
& -\frac{\mathrm{d}}{\mathrm{d} \Lambda} V=V^{2} \frac{\Lambda^{2} \rho_{0}}{{\sqrt{\Lambda^{2}+\Sigma^{2}}}^{3}}-3 V^{2} \Sigma\left(-\frac{\mathrm{d} \Sigma}{\mathrm{d} \Lambda}\right) \int_{\Lambda}^{W} \mathrm{~d} \xi \frac{\xi^{2} \rho_{0}}{E^{5}} \\
& -\frac{\mathrm{d}}{\mathrm{d} \Lambda} \Sigma=V \Sigma \rho_{0} \frac{1}{\sqrt{\Lambda^{2}+\Sigma^{2}}} .
\end{aligned}
$$

Notice that we have replaced the usual derivative with respect to $s$ by $-\Lambda \mathrm{d} / \mathrm{d} \Lambda$. The typical shapes of this flow are exhibited in Fig. 目 We see that the divergence of the effective interaction is regularized by the initial symmetrybreaking field. Hence, we can integrate out all modes of the fermionic spectrum. The four-point function in the CDW problem behaves analogously to the linear combination of normal and anomalous four-point functions in the BCS problem [7] which drives the flow of the pairing amplitude. The $\Lambda$-dependence of $\Sigma$ around the scale where $V$ peaks approaches a kink for $\Delta_{\text {ext }} \rightarrow 0$. The graph of $\Sigma(\Lambda)$ resembles the graph of $\Sigma(T)$, showing that temperature acts in a similar fashion as the cutoff in this system. The main difference between the graphs is that $\Sigma(T \rightarrow 0)$ saturates exponentially while $\Sigma(\Lambda \rightarrow 0)$ is linear.

The impact of the self-energy feedback on the RG flow can be thoroughly understood by analyzing the terms appearing in (14) and (15). Bear in mind that we think of the flow as progressing from larger to smaller values of $\Lambda$, i.e. from right to left in our plots. We first consider the limit
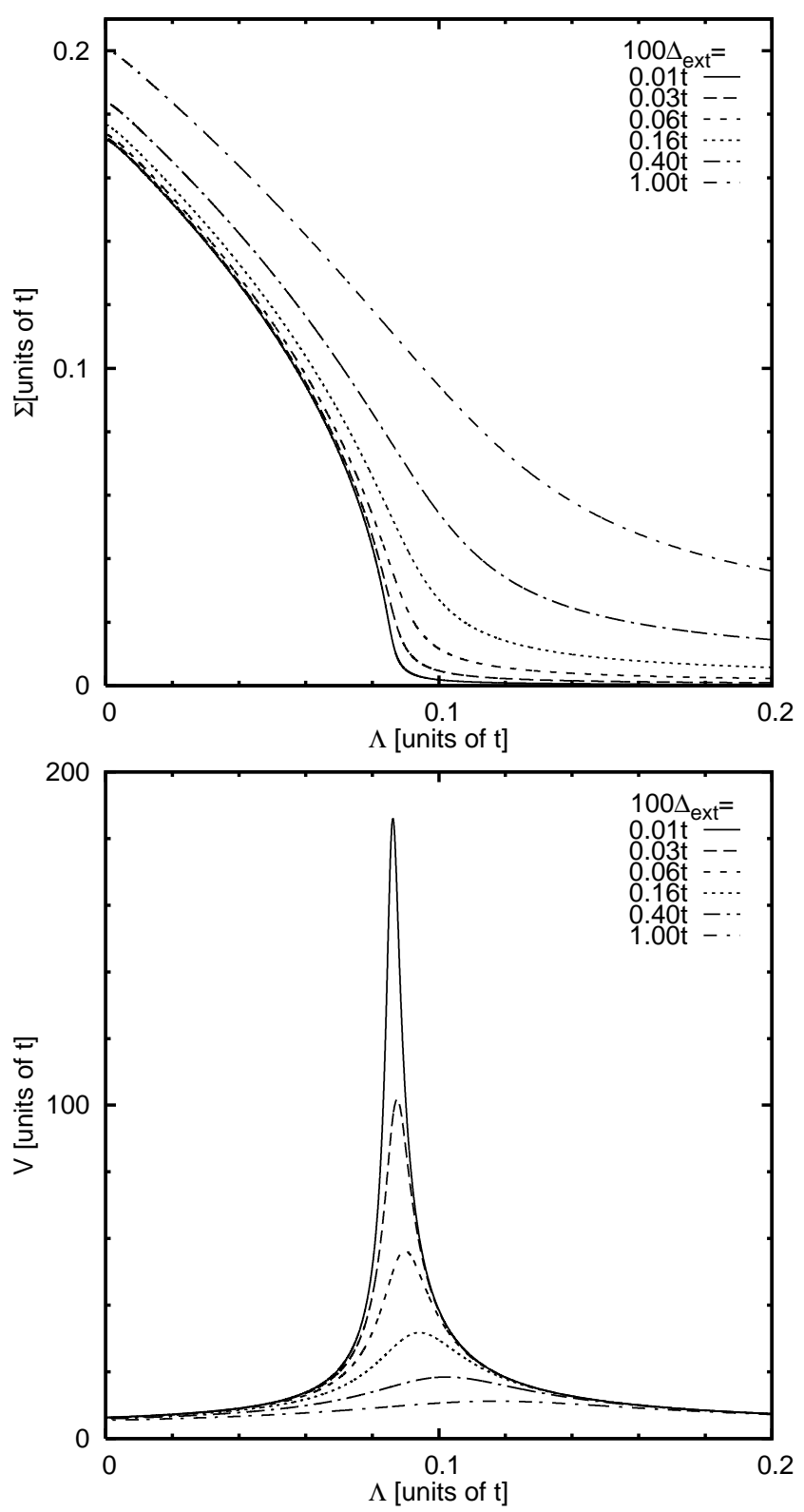

Fig. 4. Low-energy portions of the $T=0$ flows of the selfenergy (top) and effective interaction (bottom) for $V_{0}=2 t$ and various $\Delta_{\text {ext. }} \rho_{0}=1 / 2 \pi$ for all numerical calculations. Compare with Fig. 2

$\Sigma=0$. Now, the self-energy no longer flows while the effective interaction flows according to $-\mathrm{d} V / \mathrm{d} \Lambda \propto V^{2} / \Lambda$. For any positive $V_{0}$, the solution of the corresponding initial value problem is singular, showing that at $T=0$ symmetry is broken for all repulsive initial interactions.

An arbitrarily small initial symmetry-breaking field immediately has dramatic consequences: As soon as $V$ begins to increase strongly, so does the self-energy due to the coupled effects of a large $V$ and a back-feeding $\Sigma$ on the RHS of (15). The second term on the RHS of (14) is negative. It corresponds to a correction of the flow by the modes which have been integrated over at $\Lambda^{\prime}=\xi>\Lambda$ reflecting that their self-energy $\Sigma$ is changing in the flow. 
It becomes large as the slope of $\Sigma$ exceeds $\Sigma$ itself, while the positive first term is damped by an effective $\Lambda^{2} / \Sigma^{3}$ dependence as soon as $\Sigma$ becomes much larger than $\Lambda$. The effective interaction is hence pulled back down. It never reaches zero since the negative second term on the RHS of (14) becomes proportional to $V^{3}$ while the positive first term becomes proportional to $V^{2}(\mathrm{~d} \Sigma / \mathrm{d} \Lambda$ is $O(V))$. This implies that the self-energy never decreases. The two terms on the RHS of (14) thus approach mutual cancellation, causing $V$ and in turn $-\mathrm{d} \Sigma / \mathrm{d} \Lambda$ to be almost constant in $\Lambda$ (the contributions from $\Sigma$ on the RHS of (15) cancel when $\Sigma \gg \Lambda$ ).

The behavior described above remains qualitatively the same at finite temperatures, as long as these are below the double width at half maximum of $V^{\Delta_{\text {ext }}}(T)$. This is illustrated by the lowest-temperature curve of the lower panel of Fig. [5 The shape of $V(\Lambda)$ is very similar to the shape of $V(T)$ in the lower part of Fig. 2 As it is found in many one-dimensional problems, temperature thus has an effect comparable to that of an energy or momentum cutoff, respectively, as implemented here.

\section{Renormalization Group at Finite Temperatures}

We now turn to the analysis of the finite temperature fRG equations (12) and (13). Due to their more involved nature, the analysis is largely numeric. We find the same behavior as calculated using the conventional resummation methods applied in section 3. The initial symmetrybreaking field plays the same crucial role as in the zerotemperature case. We use a coupling of $V_{0}=2 t$ in the following.

The upper plot of Fig. 5 shows how the flow of the self energy flattens out when the temperature is increased beyond $T \approx 0.1 t$. Above $T_{c}, \Sigma(\Lambda=0)$ vanishes in the limit $\Delta_{\text {ext }} \rightarrow 0$. The RG flow of the effective interaction is shown in the lower panel of Fig. 5] The graph of the flow is pushed to the left with increasing temperature, its shape remaining largely unchanged. As the maximum approaches zero, the final value of the effective interaction increases until the maximum has reached $\Lambda=0$. This corresponds to the behavior of the effective interaction on the low-temperature side of Fig. 2 (lower part). For even higher temperatures, the final value of the effective interaction decreases, corresponding to the behavior on the high-energy side of Fig. 2

The lower part of Fig. 6 shows that $\Lambda_{c}(T)$ saturates quickly far below the critical temperature. It also illustrates the motion of the effective interaction flow maximum, which is pushed to lower scales by an increase in temperature as described above. It approaches zero in a linear fashion with increasing temperature, in contrast to $\Sigma\left(T \rightarrow T_{c}\right)$, which exhibits a square-root behavior.

We are furthermore interested in the dependence of the flow of the self-energy at finite temperatures on $\Delta_{\text {ext }}$. In contrast to the resummation treatment case (see the upper part of Fig. 2), we cannot set $\Delta_{\text {ext }}$ to 0 as the effective interaction would diverge before the flow reaches
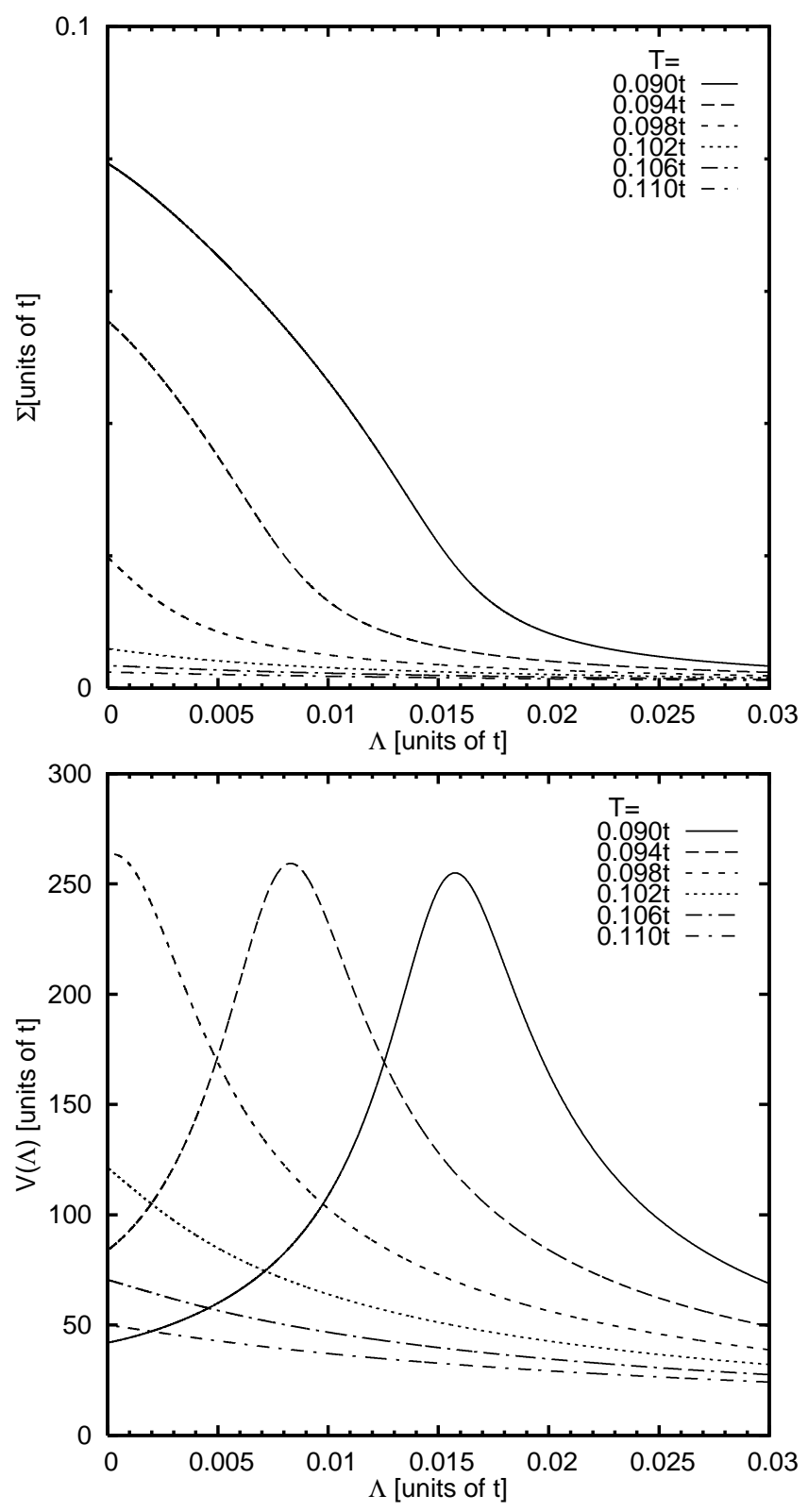

Fig. 5. Flows of the self-energy $\Sigma$ and the effective interaction $V$ plotted against $\Lambda$ for temperatures around the transition, $V_{0}=2 t$ and $\Delta_{\text {ext }}=10^{-4} t . \rho_{0}=1 / 2 \pi$ for all numerical calculations.

zero scale. However, the upper part of Fig. 4 implies that $\lim _{\Delta_{\text {ext }} \rightarrow 0} \Sigma_{\Delta_{\text {ext }}}(\Lambda)$ exists and is approached in a continuous fashion.

For $T>0$, if $\Delta_{\text {ext }}$ is one or two percent of the final $\Sigma$, there is a clearly observable steep rise (see the upper part of Fig. 5 which would allow a rather precise determination of $T_{c}$ even in more complicated models. However, note that for these values of $\Delta_{\text {ext }}$, the effective interaction at the critical scale still reaches values $\sim 100 t$ which are much larger than the bandwidth $4 t$ (see, e.g., the lower part of Fig. 7 for the temperature evolution). The agreement between $\mathrm{fRG}$ and mean-field results despite the fact that the effective interaction has grown to values large enough 

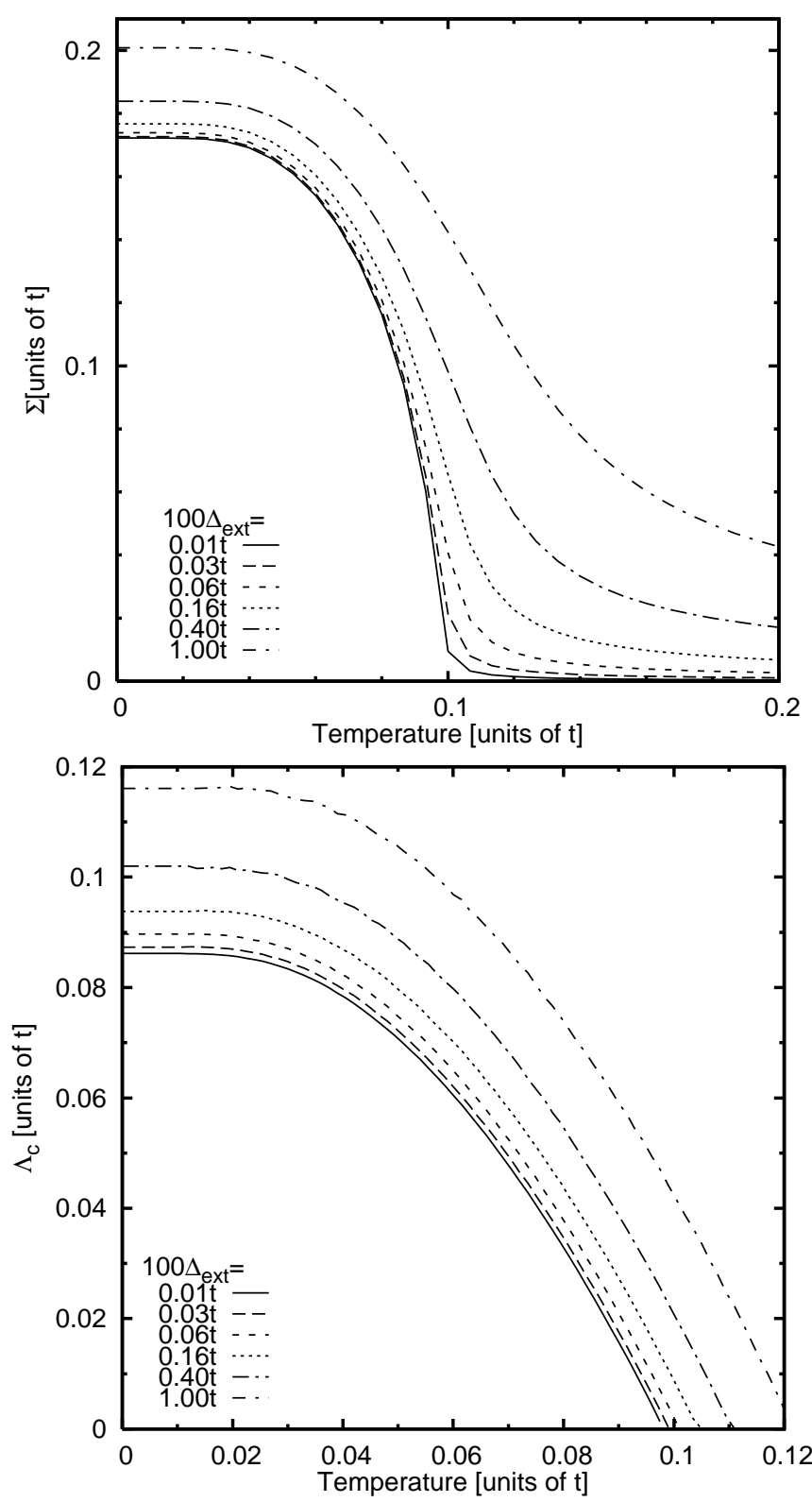

Fig. 6. (top) Self-energy against temperature calculated using fRG methods for $V_{0}=2 t$ and small to intermediate $\Delta_{\text {ext }}$. Minute deviations in comparison to Fig. 2 are due to numerical errors in the integration of the fRG differential equations. (bottom) Position of the maximum of the cutoff-dependent effective interaction against temperature for small to intermediate $\Delta_{\text {ext }}$. $\rho_{0}=1 / 2 \pi$ for all numerical calculations.

to render any perturbative scheme useless in any general model underlines that the truncated fRG is exact for our model.

At the $T$-dependent critical scale $\Lambda_{c}$, the effective interaction reaches a maximum whose height depends singularly on $\Delta_{\text {ext }}$ (see Fig. 17). A numerical analysis shows that the maximal effective interaction is $\propto 1 / \Delta_{\text {ext }}^{\alpha}$ with $\alpha \approx 2 / 3$. The values for the maximal effective interaction can be read off for $T=0$ in Fig. 团 (bottom) and for finite temperatures in the lower part of Fig. (7 For a straightfor- ward application of the method to non-mean-field models, it may be necessary to restrict the maximum of the effective interaction to smaller values to justify a perturbative treatment. Then the rise of $\Sigma_{\Lambda=0}(T)$ at $T_{c}$ gets smeared out strongly (see Fig. [6 (upper part) and Fig. 2 (upper part)). The upper part of Fig. [ 7 shows that far below $T_{c}$, the relative error $\Delta \Sigma / \Sigma$ is linear in $\Delta_{\text {ext }}$. At the highest considered $\Delta_{\text {ext }}$, it is already of the order of 0.1 while $V\left(\Lambda_{c}\right)$ is still larger than two times the bandwidth.
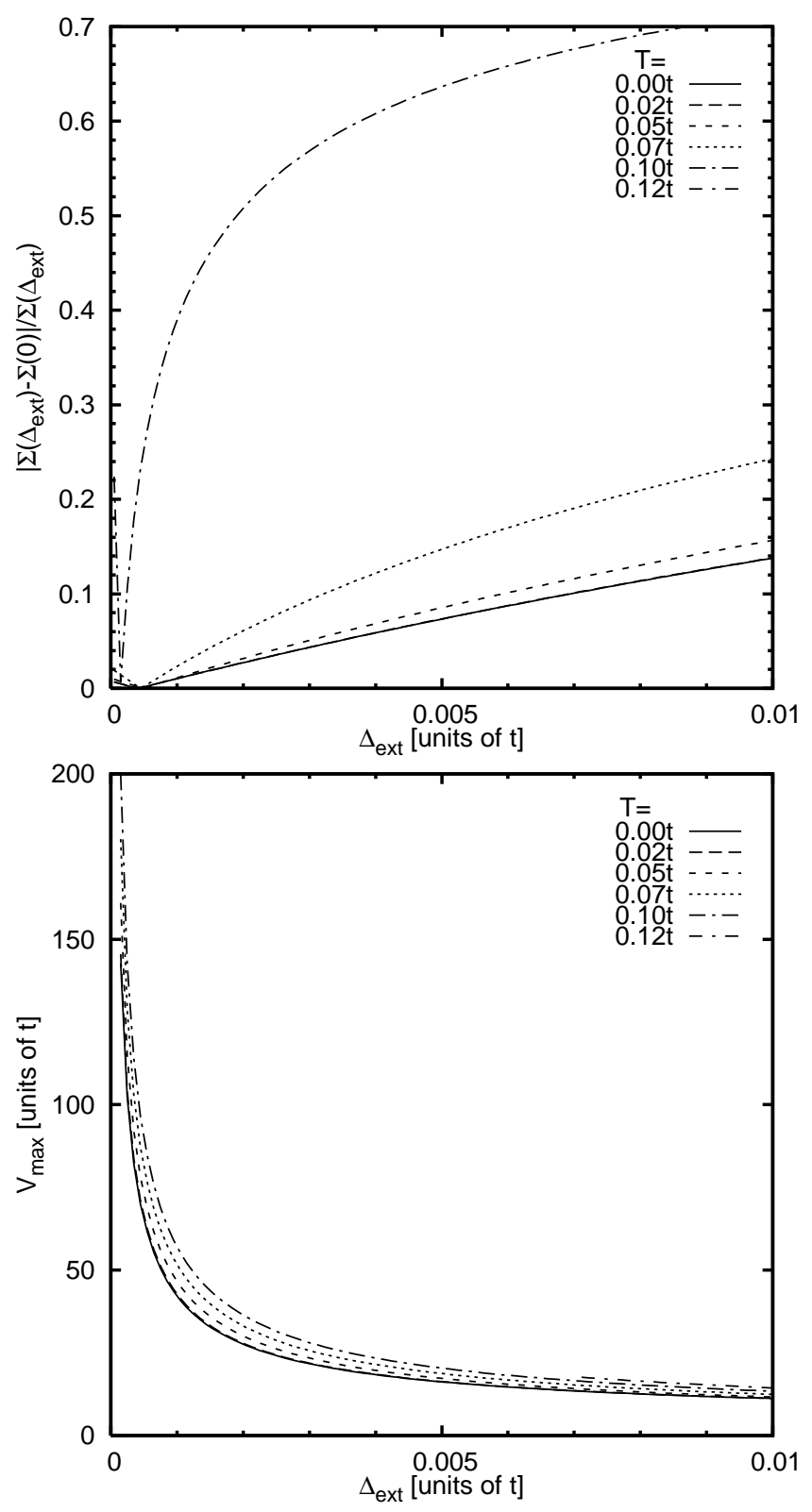

Fig. 7. (top) Relative deviation of the fRG-derived self-energy from the value without external field against the initial anomalous self-energy for temperatures from $T=0$ to just above $T_{c}$. The values for $T=0.12 t$ are 1 and thus not visible in the selected range. (bottom) Maximal value of the effective interaction during the flow against the initial anomalous self-energy for temperatures from zero to just above $T_{c}$. 


\section{Conclusion}

We have applied the functional renormalization group to a simple model for the CDW transition of spinless fermions on a hypercubic lattice in $d$ spatial dimensions. The main purpose was to test and further understand the recently developed method for the fRG flow into symmetry-broken phases in the case of a broken discrete symmetry and to generalize it to finite temperatures.

Earlier RG treatments of low-dimensional interacting fermions generically encountered flows to strong coupling where a class of coupling constants becomes too large to be treatable perturbatively. Then, the flow had to be stopped. While these flows still contain ample information about the low-energy physics of the system, a continuation down to lowest scales is desirable to obtain a controlled access to possible symmetry-broken phases. A recent treatment of the BCS model showed that the divergence of the interactions at finite RG scale can be avoided by introducing a small initial symmetry-breaking field. Furthermore, a correct treatment of the flow of the corresponding anomalous self-energy yields the correct value for the energy gap at zero scale. The interaction vertex corresponding to the Goldstone boson in the case of breaking of the continuous $\mathrm{U}(1)$ symmetry still becomes large $\propto 1 / \Delta_{\text {ext }}$ when the initial symmetry-breaking field $\Delta_{\text {ext }}$ is sent to zero.

Here we applied this method to the CDW problem with a half-filled band. Including a small initial anomalous CDW self-energy $\Delta_{\text {ext }} \neq 0$ we found that the flow of the effective interaction goes through a maximum at the critical scale $\Lambda_{c}$ and falls off below. In this case, the effective interaction does not become large as $\Lambda \rightarrow 0$ and no anomalous interactions are generated, reflecting the fact that the discrete symmetry breaking of the commensurate CDW does not produce a Goldstone mode. At $\Lambda_{c}$, the anomalous self-energy $\Sigma$ corresponding to the strength of the discrete symmetry breaking rises steeply from its initial value $\Delta_{\text {ext }}$ and finally approaches a finite value for $\Lambda \rightarrow 0$. Letting $\Delta_{\text {ext }} \rightarrow 0$, we found that $\Sigma_{\Lambda=0}$ converges to the exact result from the case without explicit symmetry breaking, $\Delta_{\text {ext }}=0$.

Studying the flow at nonzero temperatures, we have noted that the temperature dependence of the critical scale differs qualitatively from the temperature dependence of the offdiagonal self-energy, i.e. no square-root like rise of $\Lambda_{c}(T)$ is found below $T_{c}$. Therefore, $\Lambda_{c}(T)$ is only a rough estimate for the self-energy. Thus, a proper treatment of the flow of the self-energy into the symmetrybroken phase is needed in order to assess the temperature dependence of the spectral gap.

Similar to the BCS problem, the maximal value of the effective interaction at $\Lambda_{c}$ depends crucially on the explicit symmetry breaking $\Delta_{\text {ext }}$. For the mean-field model we have investigated in the present work, the truncated RG scheme is exact. Thus, large effective interactions do not cause problems. However, for the practical application of the fRG scheme to non-mean-field models where our truncated RG scheme is not exact it is necessary that the interactions do not become too large at any scale. Otherwise the feedback of the interactions in the ordering channel on processes in other channels would become large and the perturbative RG would break down. In the case of breaking of a discrete symmetry studied here, the only region where the interactions become large at zero temperature is near $\Lambda \approx \Lambda_{c}$. There, however, our explicit calculation shows that if we want to stay close to the meanfield results without explicit symmetry breaking we cannot choose too large a $\Delta_{\text {ext }}$. This in turn leads to maximal values of the effective interaction which are much larger than the bandwidth. If one wants to work with couplings which stay within the order of the bandwidth (or expressed differently, with dimensionless coupling less than one), the deviations from the $\Delta_{\text {ext }}=0$ results are rather large. At finite temperatures, the explicit symmetry breaking smears out the transition. Again, the onset of the growth of the off-diagonal self-energy only allows for a reasonable definition of a critical temperature when the maximal effective interaction is allowed to get large.

On the one hand, the large effective interactions discussed above could spoil the application of this truncated RG scheme to non-mean-field models. On the other hand, one should keep in mind that in non-mean-field models large effective interactions only occur for a narrow range of frequencies and momenta, if the bare interaction is weak. Hence, the feedback of these large interactions into other interaction channels will be strongly reduced by phasespace factors. It will be interesting to see how this physics saves or destroys for more general types of interactions the mean-field picture of the reduced models. In particular, the space dimension plays a crucial role in this respect. Understanding these issues could provide insights into the emergence of short-range ordered states in a weak coupling picture.

With the steps outlined in this article, we believe that the fRG flow into symmetry-broken states is well-understood for mean-field-type models. In the future it can be generalized to more complicated models, where it will reveal its full usefulness. Finally, we note that an alternative method for the flow into the symmetry-broken phase based on a two-particle irreducible RG formalism has been put forward by Dupuis [17. It will be interesting to explore the applicability of these new RG schemes in more realistic models.

We thank Tilman Enss, Andrey Katanin, Julius Reiss and Manfred Salmhofer for useful discussions.

\section{References}

1. M. Salmhofer and C. Honerkamp, Prog. Theor. Phys. 105, 1 (2001).

2. W. Metzner, C. Castellani, and C. Di Castro, Adv. Phys. 47, 317 (1998).

3. R. Shankar, Rev. Mod. Phys. 66, 129 (1994).

4. J. Reiss, D. Rohe, and W. Metzner, to be published (2005).

5. T. Baier, E. Bick, and C. Wetterich, Phys. Rev. B 70, 125111 (2004).

6. A. Läuchli, C. Honerkamp, and T. M. Rice, Phys. Rev. Lett. 92, 037006 (2004).

7. M. Salmhofer, C. Honerkamp, W. Metzner, and O. Lauscher, Prog. Theor. Phys. 112, 943 (2004). 
8. B. Mühlschlegel, J. Math. Phys. 3, 522 (1962).

9. P. A. Lee, T. M. Rice, and P. W. Anderson, Solid State Commun. 14, 703 (1974).

10. M. J. Rice and S. Strässler, Solid State Commun. 13, 125 (1973).

11. J. Bardeen, L. N. Cooper, and J. R. Schrieffer, Phys. Rev. 108, 1175 (1957).

12. D. Jérome, T. M. Rice, and W. Kohn, Phys. Rev. 158, 462 (1967).

13. G. Grüner and A. Zettl, Phys. Rep. 119, 117 (1985).

14. C. Wetterich, Phys. Lett. B 301, 90 (1993).

15. A. A. Katanin, Phys. Rev. B 70, 115109 (2004).

16. T. Morris, Int. J. Mod. Phys. A 9, 2411 (1994).

17. N. Dupuis, cond-mat/0506542 (2005). 